\title{
RECONSTRUCTION OF SCIENTIFIC INVESTIGATION IN INDONESIA
}

\author{
Teguh Prihmono \\ Indonesian Police Academy \\ prihmon@gmail.com
}

\begin{abstract}
The purpose of this study is to analyze the process of scientific investigation, the empowerment of scientific investigation by investigators, obstacles faced and finding scientific investigation based ideal of justice. This study uses empirical juridical approach that is related to the professional investigators and forensic laboratory examiner in conducting scientific investigations, also used a qualitative approach of the source of primary data and secondary data, then analyzed diskriftif with sestematika sentence further discussion is concluded. The problem is analyzed with proof theory and the theory of legal certainty so that it can be concluded find scientific investigation based on the ideal of justice.
\end{abstract}

Keywords: Confidence judge, Reconstruction, Scientific Investigation,

\section{A. INTRODUCTION}

Indonesia adopts a negative proofment (Negatief Wettelijke Bewijs Theorie) ${ }^{1}$, it is a verification system that is based on the evidence that has been determined by the law and the judge's conviction in giving its decision on proven or not proven fault which the accused defendant. The verification system negatively regulated in the Criminal Procedure Code article 183 "The judge must not convict to one unless at least two legal evidence, he gained confidence that a crime actually occurred and that the defendant is guilty doing it." ${ }^{\prime 2}$ Tool valid proof article 184 of the Criminal Code is the expert testimonies, letters, instructions, statements of witnesses and the accused. Thus the judge in imposing punishment should be at least two items of evidence on the five items of evidence set out in article 184 of the Criminal Procedure

1 Moeljatno. 2000, Principles of Criminal Law, Rineka PT Cipta, Jakarta, p 54.

2 Law of the Republic of Indonesia Number 8 of 1981 on Criminal Proceedings.
Code. Evidence obtained from investigators from the crime scene and other places related to the criminal case, the acquisition of evidence from the crime scene for further investigation process / sheave into evidence through scientific principles supported by the Forensic Laboratory as the main pillar supporting the investigation by whose contribution in the form of scientific evidence and expert testimony documentary evidence, that evidence of its two other objective case with the evidence in the form of instructions, witness testimony and the testimony of the defendant that is susceptible to subjective, so that when the investigators carry out scientific investigations it will obtain objective results thus will increase the confidence of judges. However, there are investigators who are less empowered scientific investigation, this is due to various factors. From the description the problem can be formulated; how the process of scientific investigation, whether the investigators had empower scientific investigation with the maximum and how 
the constraints faced and why the investigation have not been scientifically ideal that needs reconstruction. This study uses how the process of scientific investigation, whether the investigators had empower scientific investigation with the maximum and how the constraints faced and why the investigation have not been scientifically ideal that needs reconstruction. This study uses how the process of scientific investigation, whether the investigators had empower scientific investigation with the maximum and how the constraints faced and why the investigation have not been scientifically ideal that needs reconstruction. This study uses juridical empirical approach. This approach is used because of issues to be addressed relating to professional investigators and inspectors Police Forensic Laboratory in the implementation of scientific investigation. Juridical empirical approach is used with the hope to obtain a picture that is clear and intact on the background and details of the implementation of the inquiry scientifically by the police, as well as to determine the constraints faced in the implementation of the inquiry scientifically and find construction investigation scientificallybased justice ideal, it also uses a qualitative approach of primary and secondary data sources were analyzed descriptively with a systematic discussion next sentence concluded. The problem is analyzed with the theory of evidence in accordance with the verification system adopted in Indonesia and the theory of legal certainty by legislation which contains general rules to provide guidance to individuals to behave in society, both in relationships with other people and in relation to society. ${ }^{3}$ It can be concluded find scientific investigation based justice because it can contribute to the magistrate judge of conviction intact so that in deciding criminal cases do not hesitate.

\section{B. Research Methods}

This study uses empirical juridical approach that is related to the professional investigators and forensic laboratory examiner in conducting scientific investigations, also used a qualitative approach of the source of primary data and secondary data, then analyzed diskriftif with sestematika sentence further discussion is concluded. The method of approach in this research was sociological jurisdiction. This study not only examines the norms system in the rule of law, but observes the reactions and interactions that occur when the norm system works in society. Data types used were primary and secondary data. ${ }^{4}$

\section{RESULTS AND DISCUSSION}

\section{The process of scientific investigation}

The process of scientific investigation of the role of forensic laboratories.Police Forensic Laboratory as a supporter of scientific investigation is ${ }^{5}$ :

a. At this stage of the investigation helped to process the scene to

3 Peter Mahmud Marzuki, 2008, Introduction to Law, Kencana, Jakarta, p. 158.

4 Said Gunawan, Anis Mashdurohatun, Teguh Prasetyo, I Gusti Ayu Ketut Rachmi Handayani, Development Concept of Non-Alutsista Abuse By Indonesian National Army, International Journal of Business, Economics and Law, Vol. 13, Issue 4 August 2017.p. 180

5 Interview with KBP Dr. Nursamran Subandi, M.Si, Head of the Laboratory Forensi Semarang Branch, Friday, July 6, 2018. 
determine the case is a criminal act or not.

b. At this stage of the investigation, if the investigation of the incident was a criminal act, then the event / upgraded to the investigation of the case. Labfor role in the investigation process contributed to the search and collection of evidence (BB) at the crime scene (the scene). BB collected after the next process scientifically examined so that the BB can talk through the mail in the form of the minutes of the examination results.

c. Labfor examination results can be used as a development case.

d. Labfor examination results can be used as evidence is a letter / statement of experts.

\section{Supporting data of Police} Forensic Laboratory as a supporter of scientific investigation is as follows:

Table 1

Tool - special tools for the inspection of goods proof in support of scientific investigation

\begin{tabular}{|c|c|c|c|}
\hline NO & $\begin{array}{c}\text { NAME FOR } \\
\text { MAINTAINING }\end{array}$ & USE & $\begin{array}{c}\text { WORK } \\
\text { PRINCIPLE }\end{array}$ \\
\hline 1 & $\begin{array}{l}\text { Gas Cromatography } \\
\text { (GC) }\end{array}$ & $\begin{array}{l}\text { Examination of } \\
\text { evidence: Drugs, } \\
\text { fire, explosive } \\
\text { high explosive, } \\
\text { toxic material } \\
\text { unknown but it } \\
\text { should be with a } \\
\text { comparator }\end{array}$ & $\begin{array}{c}\text { Decompose by } \\
\text { heating the metal } \\
\text { (need } \\
\text { comparison) }\end{array}$ \\
\hline 2 & $\begin{array}{c}\text { Gas Cromatography } \\
\text { Mass } \\
\text { Spectrophotometer } \\
\text { (GC-MS) }\end{array}$ & $\begin{array}{l}\text { Examination of } \\
\text { evidence: } \\
\text { unknown } \\
\text { materials, } \\
\text { poisons, drugs, } \\
\text { fire, explosive } \\
\text { high explosive. }\end{array}$ & $\begin{array}{l}\text { Decompose by } \\
\text { heating in } \\
\text { columns and in } \\
\text { combination with } \\
\text { molecular weight } \\
\text { (without } \\
\text { comparison) }\end{array}$ \\
\hline
\end{tabular}

\begin{tabular}{|c|c|c|c|}
\hline NO & $\begin{array}{c}\text { NAME FOR } \\
\text { MAINTAINING }\end{array}$ & USE & $\begin{array}{c}\text { WORK } \\
\text { PRINCIPLE }\end{array}$ \\
\hline 3 & $\begin{array}{c}\text { Atomic Absorbance } \\
\text { Spectrophotometer } \\
\text { (AAS) }\end{array}$ & $\begin{array}{l}\text { Examination of } \\
\text { heavy metals } \\
\text { such as cases of } \\
\text { environmental } \\
\text { pollution, } \\
\text { poisoning, } \\
\text { explosives, etc. }\end{array}$ & $\begin{array}{l}\text { Determining the } \\
\text { concentration of } \\
\text { heavy metals by } \\
\text { the excitation } \\
\text { energy is } \\
\text { absorbed }\end{array}$ \\
\hline 4 & $\begin{array}{l}\text { Ion Cromatography } \\
\text { (IC) }\end{array}$ & $\begin{array}{l}\text { Examination of } \\
\text { cations and } \\
\text { anions, organic } \\
\text { acids, amino } \\
\text { acids, } \\
\text { explosives, } \\
\text { environmental } \\
\text { and toxicology / } \\
\text { poisoning }\end{array}$ & $\begin{array}{l}\text { Decomposition of } \\
\text { the compound in } \\
\text { the form of } \\
\text { cations and } \\
\text { anions by heating } \\
\text { the column }\end{array}$ \\
\hline 5 & $\begin{array}{c}\text { Video Spectral } \\
\text { Comparator } \\
\text { (VSC) }\end{array}$ & $\begin{array}{c}\text { Examination of } \\
\text { documents and } \\
\text { counterfeit } \\
\text { money }\end{array}$ & $\begin{array}{c}\text { Based games UV, } \\
\text { IR translucent, } \\
\text { etc. }\end{array}$ \\
\hline 6 & $\begin{array}{l}\text { Gas Cromatpgraphy } \\
\text { Infra Red (GC-IR) }\end{array}$ & $\begin{array}{l}\text { Examination of } \\
\text { evidence: } \\
\text { unknown } \\
\text { materials, } \\
\text { poisons, drugs, } \\
\text { fire, explosive } \\
\text { high explosive }\end{array}$ & $\begin{array}{l}\text { Decompose by } \\
\text { heating the } \\
\text { column and } \\
\text { combined with } \\
\text { fungsionilnya } \\
\text { group (without } \\
\text { comparison) }\end{array}$ \\
\hline 7 & $\begin{array}{l}\text { Comparison } \\
\text { Microscope }\end{array}$ & $\begin{array}{l}\text { Examination of } \\
\text { evidence: } \\
\text { projectiles, } \\
\text { bullets, shell } \\
\text { casings, tool } \\
\text { marks, etc. (by } \\
\text { comparison) }\end{array}$ & $\begin{array}{c}\text { Based on the } \\
\text { enlargement of } \\
\text { the object using a } \\
\text { lens (microscope) } \\
\text { with a } \\
\text { comparator }\end{array}$ \\
\hline 8 & $\begin{array}{c}\text { High Performance } \\
\text { Liquid Cromatography } \\
\text { (HPLC) }\end{array}$ & $\begin{array}{l}\text { Examination of } \\
\text { Drugs, } \\
\text { counterfeit } \\
\text { medicine, fuel, } \\
\text { unknown } \\
\text { materials, etc. } \\
\text { (broken at high } \\
\text { temperatures) }\end{array}$ & $\begin{array}{c}\text { The same } \\
\text { principle with } \\
\text { cromatography } \\
\text { but able at a } \\
\text { lower } \\
\text { temperature } \\
\text { because no pump }\end{array}$ \\
\hline 9 & $\begin{array}{c}\text { Total Organic Carbon } \\
\text { (TOC) }\end{array}$ & $\begin{array}{l}\text { Examination of } \\
\text { evidence in the } \\
\text { case of } \\
\text { environmental } \\
\text { pollution (total } \\
\text { carbon) }\end{array}$ & $\begin{array}{l}\text { Detect total } \\
\text { carbon }\end{array}$ \\
\hline 10 & $\begin{array}{c}\text { fluorescense } \\
\text { Spectrophotometer }\end{array}$ & $\begin{array}{l}\text { Examination of } \\
\text { compounds that } \\
\text { can ber } \\
\text { flourescense, }\end{array}$ & $\begin{array}{l}\text { Detect } \\
\text { compounds that } \\
\text { are fllourescense }\end{array}$ \\
\hline
\end{tabular}




\begin{tabular}{|c|c|c|c|}
\hline NO & $\begin{array}{c}\text { NAME FOR } \\
\text { MAINTAINING }\end{array}$ & USE & $\begin{array}{c}\text { WORK } \\
\text { PRINCIPLE }\end{array}$ \\
\hline & & drugs & \\
\hline 11 & LAB-X 3000 (X-Rays) & metal inspection & $\begin{array}{l}\text { Irradiation with } \\
\text { X-rays, excited }\end{array}$ \\
\hline 12 & $\begin{array}{l}\text { Lie Detector } \\
\text { (Polygraph) }\end{array}$ & Test lie & $\begin{array}{c}\text { Examination } \\
\text { based on heart } \\
\text { rate, perspiration }\end{array}$ \\
\hline 13 & $\begin{array}{l}\text { EMIT Solaris } \\
\text { (Immunoassay) }\end{array}$ & $\begin{array}{l}\text { Examination of } \\
\text { the urine and } \\
\text { blood drug }\end{array}$ & Spectrofotometry \\
\hline 14 & Flash Point Tester & $\begin{array}{l}\text { The discovery } \\
\text { Flash point }\end{array}$ & $\begin{array}{c}\text { Measurement of } \\
\text { the temperature } \\
\text { (flash point) }\end{array}$ \\
\hline 15 & Reprostar & $\begin{array}{c}\text { Examination of } \\
\text { documents, TLC, } \\
\text { and } \\
\text { documentation } \\
\text { management }\end{array}$ & $\begin{array}{c}\text { The camera and } \\
\text { light }\end{array}$ \\
\hline 16 & Polarimeter & $\begin{array}{c}\text { Examination of } \\
\text { polar compounds } \\
\text { rotating field }\end{array}$ & $\begin{array}{l}\text { Examination of } \\
\text { compounds that } \\
\text { are able to rotate } \\
\text { the polar field }\end{array}$ \\
\hline 17 & Radio Active Detector & $\begin{array}{l}\text { Examination of } \\
\text { radioactive } \\
\text { elements }\end{array}$ & $\begin{array}{l}\text { Detecting } \\
\text { radioactive } \\
\text { elements }\end{array}$ \\
\hline 18 & Explosive Detector & $\begin{array}{l}\text { High explosive } \\
\text { examination of } \\
\text { environmental } \\
\text { pollution at the } \\
\text { scene }\end{array}$ & $\begin{array}{l}\text { Detecting } \\
\text { explosive high } \\
\text { explosive }\end{array}$ \\
\hline 19 & $\begin{array}{l}\text { Ultra Violet Visible } \\
\text { Spectrophotometer } \\
\text { (UV-VIS) }\end{array}$ & $\begin{array}{l}\text { Multi-function } \\
\text { inspection } \\
\text { ranging from } \\
\text { explosives, } \\
\text { drugs, fuel, } \\
\text { unknown } \\
\text { material, etc. }\end{array}$ & $\begin{array}{c}\text { Examination of } \\
\text { the compound } \\
\text { based on the } \\
\text { wavelength }\end{array}$ \\
\hline 20 & $\begin{array}{l}\text { Polymerase Chain } \\
\text { Reaction (PRC) }\end{array}$ & $\begin{array}{c}\text { DNA } \\
\text { examination }\end{array}$ & $\begin{array}{c}\text { The detection of } \\
\text { DNA types in a } \\
\text { particular locus } \\
\text { with a } \\
\text { comparator }\end{array}$ \\
\hline 21 & Ion Scany & $\begin{array}{l}\text { Detecting the } \\
\text { presence / } \\
\text { absence of } \\
\text { explosive / } \\
\text { residual } \\
\text { explosives }\end{array}$ & $\begin{array}{l}\text { Detect explosives } \\
\text { based on ions in } \\
\text { the compound }\end{array}$ \\
\hline 22 & $\begin{array}{l}\text { Gastec (Gas } \\
\text { Detector) }\end{array}$ & $\begin{array}{c}\text { Detect various } \\
\text { gases include } \\
\text { density and } \\
\text { levels in a room }\end{array}$ & $\begin{array}{l}\text { Detect toxic } \\
\text { gaseous } \\
\text { compounds }\end{array}$ \\
\hline
\end{tabular}

\begin{tabular}{|c|c|c|c|}
\hline NO & $\begin{array}{c}\text { NAME FOR } \\
\text { MAINTAINING }\end{array}$ & USE & $\begin{array}{c}\text { WORK } \\
\text { PRINCIPLE }\end{array}$ \\
\hline 23 & Tensile Machine & $\begin{array}{l}\text { Tensile testing } \\
\text { metals }\end{array}$ & $\begin{array}{l}\text { Metal pulled until } \\
\text { broken, how the } \\
\text { strength / } \\
\text { toughness of the } \\
\text { metal }\end{array}$ \\
\hline 24 & $\begin{array}{l}\text { X-Ray Diffraction } \\
\text { (XRD) }\end{array}$ & $\begin{array}{l}\text { Chemical } \\
\text { composition of } \\
\text { the metal } \\
\text { inspection }\end{array}$ & $\begin{array}{c}\text { Material shot with } \\
\text { a certain angle } \\
\text { and a certain } \\
\text { energy that will } \\
\text { appear TSB } \\
\text { analyzed Graph } \\
\text { chart .... }\end{array}$ \\
\hline 25 & $\begin{array}{l}\text { X-Ray fluorescense } \\
\text { (XRF) }\end{array}$ & $\begin{array}{l}\text { Same as above } \\
\text { (Mda) }\end{array}$ & Sda \\
\hline 26 & $\begin{array}{c}\text { Scan Electron } \\
\text { Microscope (SEM) }\end{array}$ & $\begin{array}{l}\text { Examination of } \\
\text { the molecular } \\
\text { structure of the } \\
\text { metal / metal } \\
\text { microstruktur }\end{array}$ & $\begin{array}{l}\text { The molecular } \\
\text { structure of the } \\
\text { material through } \\
\text { an electron } \\
\text { microscope } \\
\text { magnified }\end{array}$ \\
\hline 27 & ESDA & $\begin{array}{l}\text { Detection of an } \\
\text { imprint in the } \\
\text { writing of the } \\
\text { document }\end{array}$ & $\begin{array}{l}\text { Detecting the } \\
\text { former former } \\
\text { oppression in } \\
\text { writing }\end{array}$ \\
\hline 28 & Shooting Box Air & $\begin{array}{l}\text { Taking samples } \\
\text { of firing } \\
\text { projectiles from } \\
\text { firearms results }\end{array}$ & $\begin{array}{l}\text { Weapons were } \\
\text { fired into the } \\
\text { water and then } \\
\text { projectile TSB } \\
\text { medium } \\
\text { automatically } \\
\text { lifted and } \\
\text { samples taken } \\
\text { intact while the } \\
\text { finger print being } \\
\text { scratched }\end{array}$ \\
\hline 29 & FTIR / Raman & $\begin{array}{l}\text { Examination of } \\
\text { evidence in the } \\
\text { form of } \\
\text { chemicals of } \\
\text { unknown } \\
\text { character } \\
\text { (explosives, } \\
\text { drugs, toxins, } \\
\text { etc.) }\end{array}$ & $\begin{array}{c}\text { Detects molecules } \\
\text { based on a range } \\
\text { of energy } \\
\text { absorption, } \\
\text { molecules that } \\
\text { interact with light } \\
\text { will produce a } \\
\text { certain energy }\end{array}$ \\
\hline 30 & responder $\mathrm{RCI}$ & $\begin{array}{l}\text { Detect chemicals } \\
\text { both solid and } \\
\text { liquid unknown }\end{array}$ & $\begin{array}{c}\text { Detecting } \\
\text { molecular } \\
\text { spectroscopy }\end{array}$ \\
\hline
\end{tabular}




\begin{tabular}{|c|c|c|c|}
\hline NO & $\begin{array}{c}\text { NAME FOR } \\
\text { MAINTAINING }\end{array}$ & USE & $\begin{array}{c}\text { WORK } \\
\text { PRINCIPLE }\end{array}$ \\
\hline 31 & MMTD & $\begin{array}{l}\text { Examination of } \\
\text { evidence, } \\
\text { especially } \\
\text { explosives, } \\
\text { drugs, toxins, } \\
\text { etc. }\end{array}$ & $\begin{array}{l}\text { Detect molecular } \\
\text { gas ions }\end{array}$ \\
\hline 32 & POISC & $\begin{array}{l}\text { Examination of } \\
\text { evidence } \\
\text { projectiles and } \\
\text { shell casings }\end{array}$ & $\begin{array}{c}\text { Finger print } \\
\text { identification, } \\
\text { projectiles and } \\
\text { shell casings, } \\
\text { then recorded } \\
\text { base }\end{array}$ \\
\hline 33 & Image Master Solo & $\begin{array}{l}\text { For the } \\
\text { acquisition of } \\
\text { laptops, PCs, } \\
\text { hard drives }\end{array}$ & $\begin{array}{c}\text { Copy the } \\
\text { contents sector- } \\
\text { by-sector } \\
\text { bitstream of } \\
\text { digital devices }\end{array}$ \\
\hline 34 & $\begin{array}{c}\text { Pableu PD3 and } \\
\text { Doggel }\end{array}$ & $\begin{array}{c}\text { For HP } \\
\text { acquisition }\end{array}$ & $\begin{array}{c}\text { Copy the } \\
\text { contents of files } \\
\text { in the HP logically } \\
\text { (data not } \\
\text { deleted) }\end{array}$ \\
\hline 35 & UFED & $\begin{array}{c}\text { For HP } \\
\text { acquisition }\end{array}$ & $\begin{array}{c}\text { Copy the } \\
\text { contents of files } \\
\text { in the HP logically } \\
\text { (data not } \\
\text { deleted) and that } \\
\text { has been } \\
\text { removed }\end{array}$ \\
\hline 36 & $\begin{array}{c}\text { FTK (Forensic) and } \\
\text { EnCase }\end{array}$ & $\begin{array}{c}\text { Analyzing } \\
\text { akuisisidari } \\
\text { Image Master } \\
\text { Solo }\end{array}$ & $\begin{array}{c}\text { From the } \\
\text { acquisition of } \\
\text { Image Master } \\
\text { Solo, then } \\
\text { analyzed in detail } \\
\text { in accordance } \\
\text { with the relevant } \\
\text { case }\end{array}$ \\
\hline 37 & $\begin{array}{l}\text { Vox Audio Foreksik } \\
\text { System }\end{array}$ & $\begin{array}{l}\text { Examination of } \\
\text { the evidence } \\
\text { recorded voice } \\
\text { (sound analysis) }\end{array}$ & $\begin{array}{l}\text { Comparing the } \\
\text { sound recording } \\
\text { sound recording } \\
\text { of evidence by } \\
\text { comparison }\end{array}$ \\
\hline 38 & $\begin{array}{l}\text { Video Foreksik } \\
\text { System Clear ID } \\
\text { Video Focus }\end{array}$ & $\begin{array}{l}\text { Examination of } \\
\text { evidence, } \\
\text { recording the } \\
\text { motion picture / } \\
\text { video, for } \\
\text { example on the } \\
\text { side of the TV, } \\
\text { mobile phones, } \\
\text { cameras, video } \\
\text { tape recorders, }\end{array}$ & $\begin{array}{l}\text { Enrichment or } \\
\text { endhancement on } \\
\text { the video frames }\end{array}$ \\
\hline
\end{tabular}

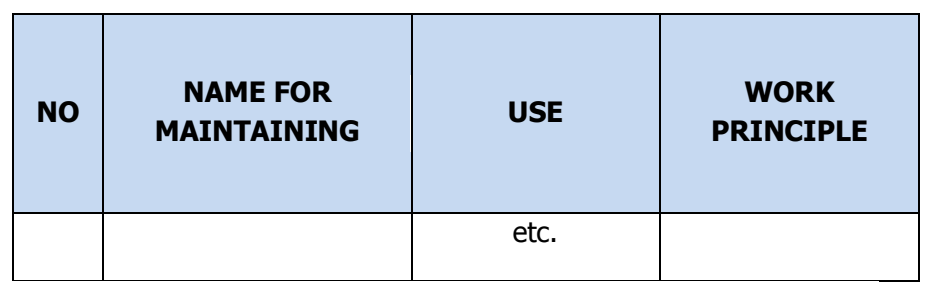

According to the authors based on data obtained mainly completeness of the instruments within their technological progress with a criminal offense that accompanies technological development, Police Forensic Laboratory as a supporter of the investigation can be scientifically prepared such that its role has been to support the investigation scientifically but still not optimally if adjusted with proof theory and the theory of legal certainty, especially instruments for DNA data base, the data base Ballistic, Digital Forensics etc ${ }^{6}$.

\section{Empowerment of scientific investigation}

a. Data examination of evidence / cases, examination of the crime scene (the scene), the expert witness and socialization that have been implemented Year period $2017^{7}$.

Table 2

Data examination of evidence / Case in Central Java Police ranks

6 Interview with Commissioner Setiawan Widiyanto, Head of Sub Division of Planning and Administration, Wednesday, July 4, 2018.

7 Interviews with the inspectors Semarang Branch Police Forensic Laboratory which Bowo Commissioner Nurcahyo, S.Si., M. Biotech, Commissioner Ibn Sutarto, ST, Commissioner Gde Buyung Dawn, ST, Adjunct Senior Commissioner Budi Santoso, S.Si., M.Sc., and Commissioner Toto Tri Kusuma, S.Si., Thursday, July 5, 2018. 


\begin{tabular}{|c|c|c|c|c|c|c|c|c|}
\hline \multirow{2}{*}{$\begin{array}{l}\text { SUBDIVISION } \\
\text { CHECKING } \\
\text { TYPE }\end{array}$} & \multicolumn{2}{|c|}{ ENTRY } & \multicolumn{2}{|c|}{ ENDS } & \multicolumn{2}{|c|}{ THE REST } & \multicolumn{2}{|c|}{$\%$} \\
\hline & $\begin{array}{c}\text { Per- } \\
\text { KAR } \\
\text { A }\end{array}$ & BB & $\begin{array}{c}\text { Per- } \\
\text { KAR } \\
\text { A }\end{array}$ & BB & $\begin{array}{l}\text { Per- } \\
\text { KAR } \\
\text { A }\end{array}$ & BB & $\begin{array}{c}\text { Per- } \\
\text { KAR } \\
\text { A }\end{array}$ & BB \\
\hline $\begin{array}{l}\text { FORENSIC } \\
\text { CHEMISTRY } \\
\text { BIOLOGY }\end{array}$ & 55 & $\begin{array}{r}2,4 \\
52\end{array}$ & 55 & $\begin{array}{r}2,4 \\
52\end{array}$ & - & - & 100 & 100 \\
\hline - Chemistry & 37 & $\begin{array}{r}2.36 \\
3\end{array}$ & 37 & $\begin{array}{r}2.3 \\
63\end{array}$ & - & - & 100 & 100 \\
\hline - Toxicology & 13 & 46 & 13 & 46 & - & - & 100 & 100 \\
\hline $\begin{array}{l}\text { - Biology / } \\
\text { Serology }\end{array}$ & 5 & 43 & 5 & 43 & - & - & 100 & 100 \\
\hline $\begin{array}{l}\text { DRUGS } \\
\text { FORENSIC }\end{array}$ & $\begin{array}{r}1,55 \\
7\end{array}$ & $\begin{array}{r}318 \\
.78 \\
6\end{array}$ & $\begin{array}{r}1,5 \\
57\end{array}$ & $\begin{array}{r}31 \\
8.7 \\
86\end{array}$ & - & - & 100 & 100 \\
\hline - Narcotics & 1.271 & $\begin{array}{r}18.4 \\
52\end{array}$ & $\begin{array}{r}1.27 \\
1\end{array}$ & $\begin{array}{r}18 . \\
452\end{array}$ & - & - & 100 & 100 \\
\hline - Psychotropic & 74 & $\begin{array}{r}59.3 \\
82\end{array}$ & 74 & $\begin{array}{r}59 . \\
382\end{array}$ & - & - & 100 & 100 \\
\hline $\begin{array}{l}\text { - Dangerous } \\
\text { Drugs }\end{array}$ & 212 & $\begin{array}{r}240 . \\
952\end{array}$ & 212 & $\begin{array}{r}240 \\
.95 \\
2\end{array}$ & - & - & 100 & 100 \\
\hline $\begin{array}{l}\text { FORENSIC } \\
\text { BALLISTIC } \\
\text { METALLURGY }\end{array}$ & 81 & $\begin{array}{r}1,5 \\
62\end{array}$ & 81 & $\begin{array}{r}1,5 \\
62\end{array}$ & - & - & 100 & 100 \\
\hline - Firearms & 12 & 573 & 12 & 573 & - & - & 100 & 100 \\
\hline - Explosives & 19 & 898 & 19 & 898 & - & - & 100 & 100 \\
\hline - Metallurgy & 50 & 91 & 50 & 91 & - & - & 100 & 100 \\
\hline $\begin{array}{l}\text { COUNTERFEIT } \\
\text { MONEY } \\
\text { FORENSIC }\end{array}$ & 53 & $\begin{array}{r}3,4 \\
19\end{array}$ & 52 & $\begin{array}{r}3.4 \\
18\end{array}$ & 1 & 1 & 98 & 100 \\
\hline signatures & 22 & 59 & 21 & 58 & 1 & 1 & 95 & 98 \\
\hline - Print Product & 6 & 60 & 6 & 60 & - & - & 100 & 100 \\
\hline $\begin{array}{l}\text { - Counterfeit } \\
\text { money }\end{array}$ & 25 & $\begin{array}{r}3,30 \\
0\end{array}$ & 25 & $\begin{array}{r}3,3 \\
00\end{array}$ & - & - & 100 & 100 \\
\hline $\begin{array}{l}\text { PHYSICS } \\
\text { COMPUTER } \\
\text { FORENSIC } \\
\text { - Detection } \\
\text { kusus }\end{array}$ & 274 & 357 & 273 & $\begin{array}{r}35 \\
6\end{array}$ & 1 & 1 & 100 & 100 \\
\hline - Audio / Video & 229 & 284 & 228 & 283 & 1 & 1 & 100 & 100 \\
\hline $\begin{array}{l}\text { - Fire / traffic } \\
\text { accidents }\end{array}$ & 45 & 73 & 45 & 73 & - & - & 100 & 100 \\
\hline & $\begin{array}{r}2,02 \\
0\end{array}$ & $\begin{array}{r}326 \\
.57 \\
6 \\
\end{array}$ & $\begin{array}{r}2.0 \\
18\end{array}$ & $\begin{array}{r}32 \\
6.5 \\
74 \\
\end{array}$ & 2 & 2 & 99.9 & $\begin{array}{r}100 . \\
0\end{array}$ \\
\hline
\end{tabular}

b. Inspection Points Genesis Case (TKP)

Examination of the scene in January to August 2017 as follows:

1) Fire House Live crime scene examination at
Kendal Police Jurisdiction (January 10th 2017)

2) Bus Fire Investigation Damri scene in Bantul police jurisdiction (January 11th 2017)

3) Tank Truck Fire scene examination at the Police Jurisdiction in Tegal (January 23, 2017)

4) TKP Open Dumping Case Examination Form B3 Mill Scale and Furnance (EAF Dust) PT. Manunggal Jaya Abadi at Kendal Police Jurisdiction (January 26th 2017)

5) Examination scene Dormitory Fire brigade $\mathrm{V}$ Ex Rt. 05 Rw. 06 Sub Ngesrep Jatingaleh Polrestabes Jurisdiction Semarang in Semarang (January 29th 2017)

6) Fire Investigation crime scene Mini Market "WE" in Jl. Werkudoro No. 101 rt. 4 rw 5 Village East Slerok City Subdistrict TegalRegional Police Law (06 February 2017)

7) Fire House Live crime scene examination in Sleman Police Jurisdiction (February 7th 2017)

8) Examination of the scene Laka KBM Bus in Karanganyar Police Jurisdiction (February 27th 2017)

9) Further examination of the crime scene in the case Laka Karanganyar Police 
Jurisdiction (March 7th 2017)

10) Fire scene examination at the Regional Office BRI Mranggen Police Law (March 13th 2017)

11) Fire scene examination Wood Factory PT. Indofarma Omicron in Purworejo Police Jurisdiction (March 20th 2017)

12) Examination of the scene Laka KBM Minibus with SPM Yamaha Vega and KBM Pick Up in Tegal Police Jurisdiction (March 22, 2017)

13) Fire scene examination Rejowinangun Market in Magelang City Police Jurisdiction (March 28th 2017)

14) Warehouse fire scene examination PT. Glory Park Marina Port of Tanjung Emas Semarang in Semarang Polrestabes Jurisdiction (March 30th 2017)

15) Murder scene examination Students Taruna Nusantara in Magelang City Police Jurisdiction (March 31, 2017)

16) Storage Warehouse fire scene Sausage and Nugget PT. Light Supreme Court in East Semarang Police Jurisdiction (April 7th 2017)

17) Fire scene Indomarco in Demak Police Jurisdiction (April 10 2017)
18) TKP explosion in Ambarawa Jago Church in Semarang Police Jurisdiction (13 April 2017)

19) Fire scene shop Chemicals Bratachem in Tegal Police Jurisdiction (May 2nd 2017)

20) Examination of the scene Noka - KBM Nosin Ditreskrimum Dump Truck in Central Java Police (May 3 2017)

21) Fire scene PT. New Work Tri Gondangrejo in Karanganyar Police Jurisdiction (May 15th 2017)

22) The death scene Tk Police Academy cadets. II Brigdatar Muhammad Adam Regiment Youth Police Academy (May 18, 2017)

23) Warehouse fire scene Indomarco in Surakarta Police Jurisdiction (May 23, 2017)

24) Fire scene Mijen Furniture Factory in Semarang Polrestabes Jurisdiction (June 12th 2017)

25) TKP Laka in Karanganyar Police Jurisdiction (June 16th 2017)

26) Fire scene Printing Klaten Klaten Police Jurisdiction (June 20th 2017)

27) Fire scene Tawang Station in Semarang Polrestabes Jurisdiction (July 1st 2017)

28) The explosion scene in Cilacap Police Jurisdiction (July 5 2017) 
29) File retrieval scene CCTV PT. Narakara Jl. Erland No. 48 Semarang in Central Java Police Ditreskrimum Jurisdiction (July 6th 2017)

30) Fire Case Trengkil Market scene in Pati Police Jurisdiction (July 8 2017)

31) Fire department store scene Phantes case in Pati Police Jurisdiction (July 10th 2017)

32) Fire Case scene Pharmacies Kimia Farma in Surakarta Police Jurisdiction (July 11th 2017)

33) Ship Fire Case scene in Pati Police Jurisdiction (July 15th 2017)

34) Fire Case scene PT. Inna Group Textile Manufacture (Intimate) in Dk / Ds Dlimas district. Flat Kab. Klaten Klaten Police Jurisdiction (July 19, 2017)

35) The explosion scene in Kebumen Police Jurisdiction (July 23 2017)

36) Examination of the scene noka - nosin Ranmor in the jurisdiction of Police Trunk (02Agustus 2017)

37) Fire scene woodshed PT. Batealit Kobeks in the district of Jepara district police jurisdiction (August 7 2017)

38) Fire scene woodshed former valet in the jurisdiction of Police Tugu Semarang (August 8, 2017)
39) Capturing the scene CCTV evidence in drug cases in homes Budi Raharjo Street West Ngesrep III No. 60 RT 01 RW 09 Srondol Kulon Semarang (August 16th 2017)

40) Fire scene Kaliwungu Market in the jurisdiction of Police Kendal (August 21, 2017)

41) Capturing the scene CCTV evidence in drug cases in the Eleven Spa \& Karaoke Puri Anjasmoro Semarang Central Java Police jurisdiction (22Agustus 2017).

c. Expert Witness / Title Case

1) Expert witness the destruction of evidence in Narcotic Drugs and Psychotropic Substances in the National Narcotics Agency of Central Java province early AKBP Ir. Sapto Sri Suhartomo, stylist Fery Eko Prasetyo, S.Si. and Pengda Tk. I Saryono (01Maret 2017)

2) Expert witness on behalf of the defendant's case firecrackers Subahan Bin (late) Amat Tahari et al. Pekalongan District Court an AKBP Rostiawan A, Amdak. Playground Tk. I Heru Budi S, Kom (March 6, 2017)

3) Expert Witnesses case of adulterated alcohol in the Bantul police Labfor Semaranga.n Branch. 
Commissioner Bowo

Nurcahyo, S. Si., M. Biotech

(March 15th 2017)

4) Expert witness terrorist case of an East Jakarta District Court AKBP Rostiawan A, Amdak. (March 15th 2017)

5) Expert witness evidence Narcotics Group I in the kind of tobacco Synthetic Zaenudun suspect bin alias Ateng Labforcab Sugiyanto in Semarang late Commissioner Ibn Sutarto, ST. (April 5 2017)

6) Computer Forensics Expert Witness Viva Revelation Candrawati defendant in Semarang Labforcab an Buyung Commissioner Gde Dawn, ST. (April 6 2017)

7) Computer Forensics Expert Witness accused Sri Asmoro Eko Nugroho als. Eko Wahid als. Eko Luis bin Sri Edi Sukarno and Lukito bin Supeno defendant in an Semarang District Court Commissioner Gde Buyung Dawn, ST. (25 April 2017)

8) Expert witness Taruna Nusantara Student Murder defendant's Andi Muhammad Ramadhan ibn Hasan Saleh Mungkid Magelang District Court Commissioner Bowo an Nurcahyo, S. Si, M. Biotech and Buyung Commissioner Gde Dawn, ST. (27April 2017)

9) Computer Forensics Expert Witness cases in the District
Court of Semarang's Commissioner Gde Buyung Dawn, ST and manager I Hugeng Purwatmadi (May 2nd 2017)

10) Expert Witness Destruction of Narcotic Drugs and Psychotropic Substances without owner in Semarang in Central Java's BNNP page AKBP Ir. Sapto Sri Suhartomo and Commissioner Ibn Sutarto, ST (May 2nd 2017)

11) Expert Witness, Forensic Document case with the defendant Damar Mardana in Klaten District Court an Adjunct Senior Commissioner Budi Santoso, S, Si, M.Si and Aipda Trihadi, SH (May 17th 2017)

12) Expert witness on behalf of the defendant's case Sumantoro Miras Als Toro in Bantul District Court Commissioner Bowo an Nurcahyo, S.Si., M.Biotech.dan Pengda I Saryono (June 5 2017)

13) Expert Witness Destruction of Narcotics form of methamphetamine (meth) weighed $500 \mathrm{gr}$ an alleged R. Aprianto Bagas Candra Dewa and methamphetamine (shabu) weighing $30 \mathrm{gr}$ an Murdwiyoko Diidit suspects in Central Java BNNP Office Jl. Block B's Madukoro Semarang AKBP Ir. Sapto Sri Suhartomo, 
Commissioner Ibn Sutarto, ST and Bripka Sugiyanta, SH (June 8th 2017)

14) Expert witness evidence Destruction of Narcotics in the Square Bung Karno's Kalirejo Ungaran Stylists Fery Eko Prasetyo, S.Si. and Bripka Sugiyanta, SH (June 19, 2017)

15) Expert witness hearing in connection with the case on behalf of the suspect Rozaq Ismail Sudarmadji Als. AJI son Bambang Sudarmadji in Semarang branch's Forensic Laboratory Buyung Commissioner Gde Dawn, ST (July 10th 2017)

16) Expert witness the trial of alleged Crime of Forgery Surat Suhadi bin Atemo Kariyo suspects in Semarang branch Forensic Laboratoryan Adjunct Senior Commissioner Budi Santoso, S. Si., M. Si. (July 24 2017)

17) Witness in the annihilation of Evidence kind Sabu in Space II Ditresnarkoba Subdit Central Java Police Jl. Tidar No. 1 SemarangStylists an Fery Eko P., S. Si and Aipda Sugiyanta, SH. (July 26th 2017)

18) Military expert witness proceedings in the case of the accused Budi Hartono Koptu Nrp 31940214510373, noncommissioned officer Ramil 06 / Gemuh Kodim
0715 / Kendal in Military Trial Courtroom II-10 Semarang Jl. Ronggolawe No. 6 Semarang Westan Kompol Ibn Sutarto, ST. (July 27, 2017)

19) Technical support destruction of evidence types of methamphetamine by Central Java Police Narcotics Investigation Directorate in the Office of Central Java Police's Ditresnarkoba Aipda Sugiyanta, $\mathrm{SH}$ and manager I Dany Apriastuti. (August 9 2017)

d. Coaching Clinic to satuan region of Central Java Police / like:

1) Socialization evidence handling for inspection Labfor (Coaching Clinic) in Kebumen Police Jurisdiction (May 8, 2017)

2) Socialization evidence handling for inspection Labfor (Coaching Clinic) in Purworejo Police Jurisdiction (May 9, 2017)

3) Socialization evidence handling for inspection Labfor (Coaching Clinic) in Temanggung police jurisdiction (May 10th 2017)

4) Socialization evidence handling for inspection Labfor (Coaching Clinic) in Wonosobo Police Jurisdiction (May 16th 2017)

5) Socialization evidence handling for inspection 
Labfor (Coaching Clinic) in Magelang Police Jurisdiction (May 17th 2017)

6) Socialization evidence handling for inspection Labfor (Coaching Clinic) in Magelang City Police Jurisdiction (May 18th 2017)

7) Coaching Clinic and Tutorial Registration Process BeasiswaS2 Sebasa education and training department of Foreign Affairs in an Ipda Riana Police Adhyaksari, S.Si. (19 s / d June 22, 2017).

e. Duties and Functions Labforcab socialization Semarang:

1) Job Training Students Faculty of Mathematics and Natural Pure Chemistry, University of Diponegoro (December 20th, $2016 \mathrm{~s} / \mathrm{d}$ January 20th, 2017)

2) Job Training Student Pure Chemistry Faculty of Science and Technology Sunan Kalijaga State Islamic University in subfield of forensic drug (January $16 \mathrm{~s} / \mathrm{d}$ February 16, 2017)

3) Job Training Faculty of Biology Department of Biology, University of General Sudirman Purwokerto in Sub Chemistry forensic biology (23th January s / d February 23, 2017)

4) Survey Research Faculty of Biology Department of
Biology, University of General Sudirman Purwokerto in Sub Chemistry forensic biology (23th January s / d February 23, 2017)

5) Job Training Students of Faculty of Mathematics and Natural Sciences Department of Biology, State University of March in Sub Chemistry forensic biology (23th January s / d February 23, 2017)

6) Job Training Students of Faculty of Mathematics and Natural Sciences Department of Chemistry, Islamic University of Indonesia in the field of Drug Sub forensic (23th January s / d February 23, 2017)

7) Job Training Vocational students Jayawisata Semarang Jurasan Computer Engineering and Network Planning and Administration in Sub Semarang branch of forensic laboratories (February $1 \mathrm{~s} / \mathrm{d}$ May 1st 2017)

8) Clinical Studies Faculty of Law, University of March Surakarta in Semarang branch of forensic laboratories (May 23, 2017)

9) Visits and Field Studies Faculty of Law, University Janabadra Yogyakarta in Semarang branch of forensic laboratories (May 24th 2017) 
10) Student Research Program S1 Faculty of Biology, University of General Sudirman Purwokerto with the title: "The successful identification of Blood on Blood Sample Dry Exposed on Different Substrates and Conditions" (06 s / d June 19, 2017)

11) Field practice students of the Department of Biology, Faculty of Science and Mathematics University of Diponegoro in Sub chemistry and forensic biology Pratiwi Women's Wisdom, Mandy Yulita Irana Dewi Ayu Dwi Lestari and Hapsah Avisha. (July $10 \mathrm{~s} / \mathrm{d}$ August 10th, 2017)

12) Providing material about the Role and Function of forensic laboratories in order to expose crimes mauling in Exercise Pre Operation Jaran Temple 2017 Building Bhakti Praja Gradhika Central Java Governor's Superintendent Rostiawan Amdak. (July 24 2017)

13) Implementation of Practice Student Work Study Program S1 Faculty of Biology, University of Gadjah Mada in forensic biology chemistry SubForensic laboratorySemarang branch of an Nailah Faizah, Niken Pariwara Jatu and Nisrina Ferdowsi. (July 24 s / d August 5 2017)
14) Comparative Test Sub-field of forensic documents and counterfeit money Forensic laboratory branch to Forensic Laboratory branch Makassar an Adjunct Senior Commissioner Budi Santoso, S. Si., M. Si. and Commissioner Widiyanto Setiawan, ST., M. Si., M. Kom (July 7 2017)

15) Comparative Test Sub-field of forensic documents and counterfeit moneyForensic laboratory Semarang branch to Forensic Laboratory branch Surabaya an Adjunct Senior Commissioner Budi Santoso, S. Si., M. Si. Playground Daru Setyo W., SE. (July 26th 2017)

16) Taught courses in Criminal Forensics Youth Level III Force 50 at the Police Academy in Semarang (August 21, 2017)

17) Speakers at the public lecture for new students of the Faculty of Biology UnSoed in the Hall of the Faculty of Biology Unsoed Purwokerto an Bowo Commissioner Nurcahyo, S.Si, M.Biotech. (August 26 2017)

18) Student field practice Faculty of Science, Islamic University of Indonesia in the field of Narkobafor's Sub Latifatul Uyun and Francine Yulistiani. (August 
$8 \mathrm{~s} / \mathrm{d}$ September 8th 2017).

According to the authors based on the data obtained by the implementation of the investigation can be scientifically empowered investigators well especially the case in Narcotic Drugs and psykotropika, murder, fires, misuse of firearms, explosives, digital forensics, but there are still things that are not optimally empowerment especially the case of traffic accidents, environmental pollution, and other industrial counterfeiting eat it when adjusted for evidentiary theory and the theory of legal certainty.

\section{Obstacles encountered in the implementation scientifically-based investigation ideal justice}

a. Personnel

1) Forensic Laboratory

Personnel (Labfor); in addition still lacks the appropriate disciplines also need additional kelipatanya if later built bases Forensic Laboratory and the knowledge and skills not equally. ${ }^{8}$

2) Investigators personnel; generally investigator sedah know forensic laboratories to support investigations scientifically but there are still cases of cases can be supported but have not

8 Interview with Dr. Nursamran Subandi, M.Si, Head of the Laboratory Forensi Semarang Branch, Friday, July 6, 2018 been empowered maksiamal, for example, the case of environmental pollution, digital forensics and tertutama once the investigation of traffic accidents, it is there are several factors: ignorance, factors far from the forensic laboratory, was already able to handle yourself, use outside experts forensic laboratories, with no forensic laboratories prosecutor has received the investigative files, because they have not been socialized intensive and effective about the existence / role of forensic laboratories throughout the police investigators, have not woken bases forensic lab every regional police even in the big cities9.

\section{b. Material, facilities and services}

Conditions for maintaining (special tool) / instruments that are not upto date with limited quantity and base Laboratoium Forensic limited, so less than the maximum in support of the investigation scientifically, for example for maintaining DNA to a data base of DNA, for maintaining data base Ballistic, for maintaining the Digital Forensi and for maintaining a complete environmental pollution is also underdeveloped panggkalan

9 Interview sampling some Central Java Police investigators, Polrestabes Semarang and several Police and the Police Board of the Central Java Police, dated 9 to July 12th 2018. 
Labfor Police and city in every major city around the Indonesia. ${ }^{10}$

c. The budget

Acompassion has not supported a budget that is up for the welfare of personnel (salaries, business travel for example, came to the scene, socialization to kewilayaan, training, an expert witness Etc.), provision for maintaining, care for maintaining and building / laboratory, for a fee akriditasi, base development of forensic laboratories So dll. $^{11}$ scientific investigation implementation has not gone ideal.

d. Field of Systems and Methods

1) Less socialized Police Regulation No. 10 of 2009 to the investigator on the handling of evidence for inspection by forensic laboratories to support scientific investigation of this case due to the limited number of forensic laboratory personnel, time, and the Forensic Laboratory base so that the implementation of scientific

10 Interviews with staff planning and administration, staff the Division of Chemical and Biological Forensic Staff of the Division of Forensic Ballistics and Metallurgy and staff the Division of Physics and Computer Forensics Forensi Laboratory Branch Semarang, Friday, July 13, 2018

11 Interview with Head of the Financial Affairs and Financial Affairs StaffLaboratory Semarang branch Forensi, Friday, July 13, 2018. investigation is not running effectively and efisien. ${ }^{12}$

2) Still not up to the availability of SOP in case investigation of certain cases, for example the case in connection with the explosives, digital forensik, environmental pollution and so lain. ${ }^{13}$

\section{Construction implementation of scientifically-based investigation of the ideal of justice}
a. Personnel;
b. Material, facilities and services;
c. The budget;
d. d. Field of Systems and Methods.

\section{CONCLUSIONS}

1. The investigation process is scientifically not be separated from the Police Forensic Laboratory to support the investigation of scientific role and is very significant in contributing mainly about legal certainty, because the results scientifically in the Police Forensic Laboratory set forth in the form of Minutes of Investigation Evidence that its interests to Pro Justitia ( courts) that have legal value as evidence in the form of "letters" article 184 of the Criminal Procedure Code, if the judge still do not understand the letter then the inspectors can be present at the

12 Interviews with officers of Planning and Administrative Affairs SectionLaboratory Semarang branch Forensi, Friday, July 13, 2018.

13 Interviews with the administrative officer at the sub field of Forensic Ballistics Metallurgy, Chemical Biology and Physics Forensics Computer ForensicsForensic Laboratory Semarang branch, Friday, July 13, 2018. 
hearing to provide information on the results of the examination set forth in the letter was the result of information the examiner may have legal value as evidence is also in the form of "information specialist" therefore is a must application of investigation scientifically-based keadialan ideal.

2. Empowerment of scientific investigations involving the Police Forensic Laboratory conducted on a sub field of Forensic Ballistics Metallurgy, sub-areas of document and counterfeit money Forensics, Forensic Drug sub field, sub-field of Chemical and Biological Physics
Forensics and Computer Forensics sub-field is still not ideal.

3. Obstacles encountered in the implementation of scientificallybased investigation ideal justice is located in the field of personnel, materiel, facilities and services, budgets and systems methodology

4. Finding construction implementation scientific investigation ideal of justice based on field personnel, materiel, facilities and services, budgets and systems methodology and then to be used as a reference input to the discretion of the authorities.

\section{BIBLIOGRAPHY}

Ahmad Ali, 2012, reveals the theory of law (Legal Theory) and Theory of Justice (Judicial Prudance) including Interpretation Act (Legisprudance), Kencana, Jakarta.

Alexander Beveridge, Forensic Investigation of Explosions, Second Edition, CRC Press.

Andi Sofyan and Abd.Asis, 2014, An Introduction to Criminal procedural law, Prenada Media Group, Jakarta.

Bagir Manan, 2005, the Justice System Authoritative (a search), FH-UII Press Yogyakarta.

Clarke's Analysis of Drugs and poisons, Third Edition, Edited by Anthony C.

Eckert, WG, 1997, Introduction to forensic sciences (2nd Ed). Boston: CRC Press.

Eddy OS Hiariej, 2014, Principles of Criminal Law, Light Atma Reader, Yogyakarta.

Frank C. Barnes, Cartridges of the World, 11th Edition, By Stan Skinner Edition Gunshot.

Wounds, Practical Aspects of Firearms, Ballistics and Forensic Techniques, Second Edition, Vincent JM Di Maio. 
Today Sasangka 2007, investigations, arrests, prosecutions, and Pretrial In Theory And Practice, Forward Backward, Bandung.

Hartono, 2010, the Criminal Investigation and Law Enforcement Approach Progressive Law, Sinar Grafika, Jakarta.

Leden Marpaung 2009, Criminal Case Management Process (Research and Investigation), Mold II, Sinar Grafika, Jakarta.

Mochtar Kusumaatmadja 2006, Concepts of Law in Development, Cet. 2, Alumni, Bandung.

Muladi, 1995, Capita Selecta Criminal Justice System, the Agency Publisher Diponegoro University, Semarang.

M. Yahya Harahap, 2002, Discussion of Problems and Application of the Criminal Procedure Code, Sinar Grafika, Jakarta.

Nikolas Simanjuntak, 2009,Indonesian Criminal Procedure Law In The Circus, First edition, Publisher Ghalia Indonesia, Bogor.

Peter Rhys Lewis, Ken Reynolds, Colin Gagg, Forensic Materials Engineering Case Studies, CRC Press.

R. Susilo, the draft OF Criminal law (Penal Code) As well as the comments-comments are Complete Article Demi Article, Politeia, Bogor.

Sacipto Rahardjo, 2007, the Civilian Police Perspective Building Law, Social \& Community, Compass Book Publishers, Jakarta.

Sadjijono, 2010, Understanding the Police Law, Matter I, Laksbang Presindo, Yogyakarta.

Said Gunawan, Anis Mashdurohatun, Teguh Prasetyo, I Gusti Ayu Ketut Rachmi Handayani, Development Concept Of Non-Alutsista Abuse By Indonesian National Army, International Journal of Business, Economics and Law, Vol. 13, Issue 4 August 2017.

Tolib Effendi, 2013, the Criminal Justice System: Comparison of Components and Process Criminal Justice System In Some States, Yogyakara: Reader Yustisia.

Tongat 2008, Basics of Criminal Law Reform Cet.1 Indonesia in Perspective., UMM Press, Malang.

Yesmil Anwar \& Adang, 2008, Reform of Criminal Law, Criminal Law Reform, Grasindo, Jakarta, in 2008.

Zainuddin Ali, 2015, Islamic Law, Vol. XV, light graphics, Jakarta. 
Zulkarnaen Koto, 2011, Breakthrough Process Simplification Law in Criminal Justice, Policing Studies Journal, STIK, Jakarta.

Law No. 2 of 2002 on the Indonesian National Police.

Police Forensic Laboratory, Criminal / Forensic Laboratory, Materials Module teaching materials AKPOL 2016 